\title{
Cryo-Electron Microscopic Study of F-Pili
}

\author{
Xiong Yu ${ }^{*}$, Ying Wang ${ }^{*}$, Philip M. Silverman ${ }^{* *}$, and Edward H. Egelman ${ }^{*}$ \\ *Department of Biochemistry and Molecular Genetics, University of Virginia, Box 800733, \\ Charlottesville, VA 22908-0733, USA \\ ** Genetic Models of Disease Research Program, Oklahoma Medical Research Foundation, \\ Oklahoma City, OK 73104.
}

We used cryo-electron microscopy and single-particle methods to solve the long-existing ambiguity in the packing geometry of F-pilin subunits. Two subunit packing schemes were identified: one has stacked rings of four subunits axially spaced by $12.8 \AA$, while the other has a one-start helical symmetry with an axial rise of $\sim 3.5 \AA$ per subunit and a pitch of $\sim 12.2 \AA$. Both structures have a central lumen of $\sim 30 \AA$ diameter that is more than large enough to allow for the passage of singlestranded DNA. Remarkably, both schemes appear to coexist within the same filaments, in contrast to filamentous phages that have been described as belonging to one of two possible symmetry classes. For the segments composed of rings, the twist between adjacent rings is quite variable, while the segments having a one start helix are in multiple states of both twist and extension. Some other pili structures will also be compared and discussed. 